\title{
Avaliação do serviço de controle da tuberculose em uma unidade saúde da família no estado do Pará
}

\author{
Evaluation of the tuberculosis control service in a family health unit in the state of Pará \\ Evaluación del servicio de control de tuberculosis en una unidad de salud familiar en el \\ estado de Pará
}

Raquel Ferreira Cardoso ${ }^{1 *}$, Átila Augusto Cordeiro Pereira ${ }^{2}$, IIma Pastana Ferreira ${ }^{1}$, Claudia Ozela El-Husny ${ }^{1}$, Josias Botelho da Costa ${ }^{1}$, Bianca Silva da Cruz $^{1}$, Brenda Soele Souza Matos ${ }^{2}$, Lohanna Rafaelle Lima de Oliveira ${ }^{1}$, Sara Edyele Santos Marques², Darciane Coelho Cordovil'1.

\section{RESUMO}

Objetivo: Avaliar o serviço de controle da tuberculose em uma Unidade Saúde da Família. Métodos: Tratase de um estudo avaliativo, descritivo e quantitativo, realizado com 11 profissionais em uma Unidade Saúde da Família no estado do Pará. As entrevistas ocorreram nos meses de agosto e setembro de 2019, com aplicação de um instrumento adaptado e validado pela Rede Brasileira de Pesquisa em tuberculose. Os dados foram analisados por meio de estatística descritiva no Google Forms e Excel 2013. O estudo foi aprovado pelo Comitê de Ética em Pesquisa. Resultados: As variáveis do componente Recursos Humanos, Oferta de capacitação em TB pela atenção Básica $(54,5 \%)$ e Oferta de capacitação pelo PCT municipal $(36,4 \%)$, apresentaram desempenho insatisfatório e crítico, respectivamente. $\mathrm{Na}$ organização do serviço, apenas o Acesso ao resultado de baciloscopia em 7 dias (63,6\%) obteve um desempenho insatisfatório. $O$ componente Processo foi o que mais apresentou variáveis com desempenho crítico. Conclusão: Existem fragilidades no serviço oferecido pela Unidade, sendo necessário o estabelecimento de estratégias e a adoção da avaliação permanente como uma ferramenta na busca de melhorias da qualidade da assistência.

Palavras-Chave: Tuberculose, Estratégia saúde da família, Indicadores de qualidade em assistência à saúde.

\begin{abstract}
Objective: To evaluate the tuberculosis control service in a Family Health Unit. Methods: This is an evaluative, descriptive and quantitative study, conducted with 11 professionals in a Family Health Unit in the state of Pará. The interviews took place in August and September 2019, with the application of an instrument adapted and validated by Brazilian Tuberculosis Research Network. The data were analyzed using descriptive statistics in Google Forms and Excel 2013. The study was approved by the Research Ethics Committee. Results: The variables of the Human Resources component, Offer of training in TB through Primary care $(54.5 \%)$ and Offer of training by the municipal PCT $(36.4 \%)$, presented unsatisfactory and critical performance, respectively. In the organization of the service, only the Access to the result of smear microscopy in 7 days (63.6\%) had an unsatisfactory performance. The Process component was the one that most presented variables with critical performance. Conclusion: There are weaknesses in the service offered by the Unit, requiring the establishment of strategies and the adoption of permanent evaluation as a tool in the search for improvements in the quality of care.
\end{abstract}

Keywords: Tuberculosis, Family health strategy, Health care quality indicators.

\footnotetext{
1 Universidade do Estado do Pará (UEPA). Belém - PA. *E-mail: raquel_fcardoso@hotmail.com

2Universidade Federal do Pará (UFPA). Belém - PA.
} 


\section{RESUMEN}

Objetivo: Evaluar el servicio de control de tuberculosis en una Unidad de Salud de la Familia. Métodos: Estudio evaluativo, descriptivo y cuantitativo, realizado con 11 profesionales en una Unidad de Salud de la Familia en el estado de Pará. Las entrevistas tuvieron lugar en agosto y septiembre de 2019, con la aplicación de un instrumento adaptado y validado por Red Brasileña de Investigación de Tuberculosis. Los datos se analizaron mediante estadísticas descriptivas en Google Forms y Excel 2013. El estudio fue aprobado por el Comité de Ética en Investigación. Resultados: Las variables del componente de Recursos Humanos, Oferta de capacitación en TB a través de Atención primaria $(54.5 \%)$ y Oferta de capacitación por parte del PCT municipal (36.4\%), presentaron desempeño insatisfactorio y crítico, respectivamente. En la organización del servicio, solo el acceso al resultado de la microscopía de frotis en 7 días (63.6\%) tuvo un desempeño insatisfactorio. El componente Proceso fue el que presentó más variables con desempeño crítico. Conclusión: Existen debilidades en el servicio ofrecido por la Unidad, que requieren el establecimiento de estrategias y la adopción de la evaluación permanente como herramienta en la búsqueda de mejoras en la calidad de la atención.

Palabras clave: Tuberculosis, Estrategia de salud familiar, Indicadores de calidad de la atención médica.

\section{INTRODUÇÃO}

A tuberculose (TB) é uma doença que ainda apresenta altos índices de morbidade e mortalidade em vários países do mundo. No Brasil, caracteriza-se como um problema de saúde pública que precisa ser combatido e que tem relação direta com a pobreza (BRASIL, 2013).

Em 1998 a TB foi destacada como problema prioritário de saúde no Brasil pelo Ministério da Saúde (MS) e através de uma decisão do Conselho Nacional de Saúde (CNS) foi elaborado o Programa Nacional de Controle da Tuberculose (PNCT). Este plano engloba inúmeras iniciativas, entre elas a estratégia do tratamento diretamente observado (TDO) e a horizontalização do combate à doença para todos os níveis de complexidade do sistema único de saúde (SUS). Logo, visa à inserção da atenção básica para a expansão do acesso ao diagnóstico e tratamento da TB (BRASIL, 2017).

Apesar dos aparatos tecnológicos utilizados na área da saúde desempenharem importante papel, persistem necessidades que devem ser trabalhadas, principalmente as relacionadas ao diagnóstico, ao tratamento e acesso a medidas de promoção e prevenção (BARREIRA D, 2018). Segundo estimativas da Word Health Organization (WHO), cerca de um terço da população mundial está infectada pelo bacilo de Koch, causador da doença. Em 2017, 10 milhões de pessoas desenvolveram a TB, sendo 5,8 milhões de homens, 3,2 milhões de mulheres e 1,0 milhão de crianças. A doença atingiu todas as faixas etárias, porém sua maior população era formada por adultos (idade $\geq 15$ anos), cerca de $90 \%$ dos casos, destas, $9 \%$ eram de pessoas soropositivas (WHO, 2018).

Em 2017, no Brasil houveram 69.569 novos casos da doença notificados, 33,5 casos para cada 100 mil habitantes. Apesar dos esforços em disponibilizar o diagnóstico e tratamento eficaz no SUS, em 2016 foram notificados 4.426 óbitos por TB no país, mais de duas mortes a cada 100 mil habitantes (BRASIL, 2018a).

O maior quantitativo de casos na região norte concentra-se no Pará, no qual existe prioridade para o enfretamento da doença em sete municípios (BRASIL, 2014a). Em 2017, foram registrados no Sistema de Informação de Agravos de Notificação (SINAN), 481 óbitos na região norte, destes, 258 foram notificados no Pará (BRASIL, 2018b).

Para o enfretamento da TB faz-se necessário consolidar o PNCT, buscando avanços na prevenção e no cuidado ao paciente. Para tal, o programa propõe uma lista de indicadores que devem ser aplicados para 0 desenvolvimento de ações e monitoramento (BRASIL, 2011a). Portanto, a elaboração e monitorização das ações de vigilância tem um papel fundamental para o alcance das taxas preconizadas nos indicadores, 
principalmente o aumento da cura e diminuição do abandono (BRASIL, 2013a; BERTOLOZZI MR, et al., 2014). O PNCT recomenda a utilização de atividades de monitoramento e avaliação, proporcionando a oportunidade para orientação e motivação dos profissionais, visando ao aperfeiçoamento de habilidades e de aprendizagem, bem como a melhoria do programa com indicadores epidemiológicos e operacionais (BRASIL, 2017)

A prática de monitoramento e avaliação não é recente na saúde pública, porém tem se intensificado apenas nos últimos anos para a elaboração de propostas e ações que orientem a tomada de decisão (BRASIL, 2013). Além de ser uma ferramenta para a qualificação dos programas e serviços de saúde no âmbito da gestão (BEZERRA LCA, et al., 2009).

A avaliação deve ser realizada desde a unidade básica de saúde (UBS) até a instância central nacional, destinando-se a conhecer o grau de sucesso das metas dos programas, bem como produzir informações gerenciais para o planejamento de atividades futuras e suas eventuais modificações (BRASIL, 2011b).

Os profissionais de saúde possuem o desafio de utilizar o monitoramento e a avaliação, não somente para quantificar resultados, mas também, para identificar mudanças nas relações com as famílias e comunidade (BRASIL, 2013). Por conseguinte, o processo de avaliação do programa de controle da tuberculose (PCT) deve ser contínuo para que as unidades de saúde possam desempenhar um serviço de qualidade no cuidado à pessoa com a doença (BRASIL, 2011a).

Nesta perspectiva, a discussão da temática faz-se relevante no sentindo de, através dos indicadores específicos preconizados pelo PNCT, identificar os principais pontos que podem ser melhorados e os pontos que estão fortalecidos na assistência ao paciente, além de proporcionar, para a comunidade acadêmica e profissional, um panorama da situação do PCT em uma Unidade Saúde da Família (USF). Logo, este estudo tem por objetivo avaliar a qualidade do serviço de controle da tuberculose em uma USF, no estado do Pará.

\section{MÉTODOS}

Trata-se de um estudo avaliativo, descritivo e natureza quantitativa, realizado em um município paraense nos meses de agosto e setembro de 2019. Foram incluídos os profissionais que atuam no programa de TB em uma USF, há pelo menos 1 ano e foram excluídos da pesquisa os profissionais em gozo de férias, qualquer tipo de licença ou que se recusaram em participar. A amostragem foi composta por 11 profissionais que atuavam na USF cenário de pesquisa. A coleta de dados ocorreu após aprovação do Comité de Ética e Pesquisa (CEP) sob o número de parecer 3.404.685.

Os dados foram coletados por meio de entrevistas e aplicação de um instrumento validado pelo Grupo de Estudos Operacionais em Tuberculose (GEOTB) (SILVA DM, 2013). Foram trabalhados os indicadores de estrutura (recursos humanos, recursos físicos e organizacional) e de processo.

A entrevista com os profissionais foi realizada em local privado conforme pré- agendamento, individual, sendo explicado sobre os objetivos da pesquisa e apresentação do Termo de Consentimento Livre e Esclarecido (TCLE).

O instrumento aplicado seguiu as seguintes etapas de perguntas: Identificação do participante da pesquisa quanto formação, função exercida, tempo de atuação profissional; Questões sobre as variáveis de estrutura do serviço de TB; Questões de abordagem quanto as variáveis de processo do serviço de TB.

Os dados obtidos durante a coleta foram inseridos no Google Forms e no software Microsoft $\circledast$ Office Excel® 2013. Para aplicação da estatística descritiva foram gerados gráficos e construído as tabelas. A análise de dados se deu através da distribuição das frequências relativas e absolutas.

Foram utilizados os parâmetros descritos por Samico I, et al. (2005) e Felisberto E, et al. (2002) com interpretação de valores em: críticos $(<49,9 \%)$, insatisfatórios $(50-79,9 \%)$ e satisfatórios $(>80 \%)$, de acordo com o ponto de corte. 


\section{RESULTADOS}

Observou-se que 82\% ( $n=9)$ eram Agentes Comunitários de Saúde (ACS) e 18\% (n=2) técnicos de enfermagem. O sexo feminino representou $91 \%(n=10)$ e apenas $9 \%(n=1)$ do sexo masculino. No que diz respeito a faixa etária $37 \%(n=4)$ possuem idade entre 40 e 49 anos, $27 \%(n=3)$ entre 20 e 29 anos, 18\% $(n=2)$ possuem entre 30 e 39 anos, $9 \%(n=1)$ possuem acima de 50 anos e $9 \%(n=1)$ não respondeu à pergunta. Constatou-se que os maiores tempos de atuação na APS foram de 3 a 5 anos com $37 \%(n=4)$ e de 6 a 9 anos com $36 \%(n=4)$. Além disso, os maiores tempos de atuação na USF Santos Dumont foi entre 3 a 5 anos com $64 \%(n=7)$ (Tabela 1$)$.

Tabela 1 - Caracterização dos participantes, 2019.

\begin{tabular}{lccc}
\hline Variável & Resultados & $\mathbf{n}$ & $\%$ \\
\hline \multirow{2}{*}{ Formação profissional } & Enfermeiro & 0 & 0 \\
& Médico & 0 & 0 \\
& Agente Comunitário de Saúde (ACS) & 9 & 82 \\
Sexo & Técnico de enfermagem & 2 & 18 \\
\hline & Feminino & 10 & 91 \\
& Masculino & 1 & 9 \\
\hline Faixa etária dos participantes (anos) & $20-29$ & 3 & 27 \\
& $30-39$ & 2 & 18 \\
& $40-49$ & 4 & 37 \\
& $\geq 50$ & 1 & 9 \\
Tempo de atuação na APS (anos) & Sem resposta & 1 & 9 \\
\hline Total & 1 a 2 & 1 & 9 \\
& 3 a 5 & 4 & 37 \\
\hline Tempo de atuação na USF & 6 a 9 & 4 & 36 \\
& $\geq 10$ & 2 & 18 \\
\hline
\end{tabular}

Fonte: Cardoso RF, et al., 2020.

Os indicadores do componente estrutural recursos humanos tiveram uma variação entre $90,9 \%$ e $36,4 \%$. Observou-se que as variáveis envolvimento de outros profissionais com o atendimento aos doentes de TB (90,9\%), profissional de saúde responsável pelo TDO (90,9\%), profissionais da equipe mínima de Saúde da Família envolvidos com o atendimento aos doentes de TB (88,6\%) e capacitação para o atendimento em TB nos últimos 3 anos para auxiliar/técnico de enfermagem e ACS $(81,8 \%)$ apresentaram um desempenho considerado satisfatório (Tabela 2). Ainda neste componente, a variável oferta de capacitação em TB pelo serviço da $A B$ apresentou ponto de corte inferior a 79,9\% ficando com um desempenho insatisfatório (54,5\%). No que diz respeito a oferta de capacitação em TB pelo PCT municipal, este apresentou ponto de corte inferior a $49,9 \%$, o que refletiu um desempenho crítico em $36,4 \%$ (Tabela 2). 
Tabela 2 - Distribuição e classificação das variáveis que integram o componente estrutural (recursos humanos) do serviço de controle da tuberculose em uma USF segundo os profissionais de saúde, 2019.

\begin{tabular}{lccc}
\hline Variáveis & $\begin{array}{c}\text { Sim } \\
(\%)\end{array}$ & $\begin{array}{c}\text { Não } \\
\text { (\%) }\end{array}$ & Classificação \\
\hline $\begin{array}{l}\text { Profissionais da equipe mínima de Saúde da Família envolvidos com o } \\
\text { atendimento aos doentes de TB }\end{array}$ & 88,6 & 11,4 & Satisfatório \\
$\begin{array}{l}\text { Envolvimento de outros profissionais com o atendimento aos doentes } \\
\text { de TB }\end{array}$ & 90,9 & 9,1 & Satisfatório \\
$\begin{array}{l}\text { Capacitação para o atendimento em TB nos últimos } 3 \text { anos para } \\
\text { aux/téc de enfermagem e ACS }\end{array}$ & 81,8 & 18,2 & Satisfatório \\
Oferta de capacitação em TB pelo PCT municipal & 36,4 & 63,6 & Crítico \\
Oferta de capacitação em TB pelo serviço da AB & 54,5 & 45,5 & Insatisfatório \\
Profissional de saúde responsável pelo TDO & 90,9 & 9,1 & Satisfatório \\
\hline
\end{tabular}

Fonte: Cardoso RF, et al., 2020.

$\mathrm{Na}$ avaliação do componente estrutural recursos físicos apenas a variável acesso ao livro de registro e acompanhamento de ILTB apresentou desempenho insatisfatório (54,4\%). As variáveis acesso a ficha de notificação, acesso aos prontuários de doentes de TB, acesso ao livro de registro e acompanhamento de TB, disponibilidade de pote para baciloscopia de escarro, disponibilidade de pedido de baciloscopia de escarro, disponibilidade de pedido de exame de HIV, disponibilidade de ficha de referência e contrarreferência apresentaram um desempenho satisfatório, todos com ponto de corte de $100 \%$, seguidos do acesso a ficha diário de TDO e disponibilidade de pedido de cultura de escarro com desempenho satisfatório e ponto de corte de 90,9\% cada (Tabela 3).

Tabela 3 - Distribuição e classificação das variáveis que integram o componente estrutural (recursos físicos) do serviço de controle da tuberculose em uma USF segundo os profissionais de saúde, 2019.

\begin{tabular}{llll}
\hline Variáveis & Sim (\%) & Não (\%) & Classificação \\
\hline Acesso a ficha de notificação & 100 & 0 & Satisfatório \\
Acesso aos prontuários de doentes de TB & 100 & 0 & Satisfatório \\
Acesso ao livro de registro e acompanhamento de TB & 100 & 0 & Satisfatório \\
Acesso a ficha diário de TODO & 90,9 & 9,1 & Satisfatório \\
Acesso ao livro de registro e acompanhamento de ILTB & 54,5 & 45,5 & Insatisfatório \\
Disponibilidade de pote para baciloscopia de escarro & 100 & 0 & Satisfatório \\
Disponibilidade de pedido de baciloscopia de escarro & 100 & 0 & Satisfatório \\
Disponibilidade de pedido de cultura de escarro & 90,9 & 9,1 & Satisfatório \\
Disponibilidade de pedido de exame de HIV & 100 & 0 & Satisfatório \\
Disponibilidade de ficha de referência e contrarreferência & 100 & 0 & Satisfatório \\
\hline
\end{tabular}

Fonte: Cardoso RF, et al., 2020

Na variável estrutural que dizem respeito à organização do serviço, apenas o acesso ao resultado do exame de baciloscopia de escarro em 7 dias obteve um desempenho insatisfatório com $63,6 \%$. As demais variáveis obtiveram desempenho satisfatório, sendo a entrega de medicamentos pelo PCT e orientação quanto a busca por atendimento fora da data agendada caso necessite com os índices e 100\%, seguidos de acesso à consulta em prazo de 24 horas com $90,9 \%$ e atendimento laboratorial ao serviço com $81,8 \%$ (Tabela 4). 
Tabela 4 - Distribuição e classificação das variáveis que integram o componente estrutural (organização do serviço) do serviço de controle da tuberculose em uma USF segundo os profissionais de saúde, 2019.

\begin{tabular}{|c|c|c|c|}
\hline Variáveis & $\operatorname{Sim}(\%)$ & Não (\%) & Classificação \\
\hline $\begin{array}{l}\text { A entrega de medicamentos pelo PCT municipal atende à } \\
\text { demanda }\end{array}$ & 100 & 0 & Satisfatório \\
\hline Acesso à consulta em prazo de 24 horas & 90,9 & 9,1 & Satisfatório \\
\hline $\begin{array}{l}\text { Orientação quanto a busca por atendimento fora da data } \\
\text { agendada caso necessite }\end{array}$ & 100 & 0 & Satisfatório \\
\hline Atendimento laboratorial ao serviço atende à demanda & 81,8 & 18,2 & Satisfatório \\
\hline $\begin{array}{l}\text { Acesso ao resultado do exame de baciloscopia de escarro em } 7 \\
\text { dias }\end{array}$ & 63,6 & 36,4 & Insatisfatório \\
\hline
\end{tabular}

Fonte: Cardoso RF, et al., 2020.

O componente processo foi o que mais apresentou variáveis com desempenho crítico, sendo elas: livro de registo e acompanhamento dos doentes de TB atualizado com 45,5\%, solicitação de baciloscopia de controle mensal com $27,3 \%$, entrega de material para realização do exame de baciloscopia de controle na unidade de atendimento com $27,3 \%$ e realização teste tuberculínico (PPD) no mesmo serviço de saúde com $27,3 \%$ (Tabela 5).

Tabela 5 - Distribuição e classificação das variáveis que integram o componente processo do serviço de controle da tuberculose em uma USF segundo os profissionais de saúde, 2019.

\begin{tabular}{lccc}
\hline Variáveis & $\begin{array}{c}\text { Sim } \\
(\%)\end{array}$ & $\begin{array}{c}\text { Não } \\
(\%)\end{array}$ & Classificação \\
\hline Livro de registro e acompanhamento dos doentes de TB atualizado & 45,5 & 54,5 & Crítico \\
Regularidade no fornecimento da medicação para o doente com TB & 100 & 0 & Satisfatório \\
Orientação quanto à doença & 95,4 & 4,6 & Satisfatório \\
Orientação quanto ao tratamento & 90,9 & 9,1 & Satisfatório \\
Orientação quanto à realização do exame de HIV & 90,9 & 9,1 & Satisfatório \\
Solicitação de baciloscopia de controle mensal & 27,3 & 72,7 & Crítico \\
Entrega de material para realização do exame de baciloscopia de & 27,3 & 72,7 & Crítico \\
controle na unidade de atendimento & 100 & 0 & Satisfatório \\
Realização de consulta médica mensal no mesmo serviço de saúde & 100 & 0 & Satisfatório \\
Busca ao doente de TB quando não comparece à consulta & 100 & 0 & Satisfatório \\
Busca ao doente de TB quando não comparece à tomada da & 100 & 0 & Satisfatório \\
medicação & 27,3 & 72,7 & Crítico \\
Busca ativa dos contatos dos pacientes bacilíferos & & & \\
Realização teste tuberculínico (PPD) no mesmo serviço de saúde
\end{tabular}

Fonte: Cardoso RF, et al., 2020.

\section{DISCUSSÃO}

A partir dos resultados ficou notável a alta rotatividade de profissionais enfermeiros e médicos na USF Santos Dumont, já que os médicos e os enfermeiros de ambas as equipes tinham menos de 1 ano de serviço na referida unidade. Resultado este corroborado com uma pesquisa realizada em Belo Horizonte onde um dos maiores índices de rotatividade foi justamente dessas duas categorias profissionais (SANCHO LG, et al., 2012). Um dos fatores essenciais para o sucesso da Estratégia Saúde da Família (ESF) é a permanência dos profissionais de saúde, principalmente médicos e enfermeiros. Logo, a alta rotatividade pode acarretar danos a este modelo de atenção, interferindo na satisfação do usuário e na qualidade da assistência, pois ambos são dependentes da formação de vínculo entre profissionais e usuários (TONELLI $B Q$, et al., 2018). 
No presente estudo, constatou-se que que a maioria dos profissionais entrevistados $(63,3 \%)$ são Agentes comunitários e técnicos de enfermagem, tinham o tempo de atuação na USF entre 3 a 5 anos. Sancho LG, et al. (2012) relata que a fixação do trabalhador favorece na melhoria da qualidade da assistência, resultante da formação de vínculos entre os profissionais e a comunidade.

Em relação ao sexo, 10 (91\%) entrevistadas foram do sexo feminino e apenas 1 do sexo masculino (9\%). Lima EFA, et al. (2016) encontrou achados semelhantes em seu estudo de perfil socioprofissional de trabalhadores de equipes saúde da família. A pesquisa destacou que os profissionais de saúde, independente da categoria, são, em sua maioria, do sexo feminino.

Quanto ao componente estrutural recursos humanos o mesmo apresentou desempenho satisfatório o que demonstra o envolvimento da equipe multiprofissional com o paciente em tratamento de tuberculose. Este achado é ratificado por Rocha MA (2016), citando o ACS como um grande potencial na criação de vínculo com o usuário, além de corresponsabilizar a equipe multiprofissional no desenvolvimento de ações de saúde com a finalidade de promoção de uma assistência integral.

Ainda no componente recursos humanos, os indicadores mais prejudicados foram os relacionados à capacitação profissional em TB com desempenho insatisfatório, o que demonstra uma fragilidade na educação permanente em saúde (EPS) dos profissionais que estão envolvidos no cuidado e assistência ao paciente com tuberculose. Este achado vai de contra o que é preconizado pela Política Nacional de Educação Permanente em Saúde (PNEPS), instituída em 2004, como um instrumento de desenvolvimento e formação de profissionais do SUS (BRASIL, 2007).

Um resultado similar foi encontrado em um estudo, onde constatou-se que menos da metade dos profissionais haviam participado de ações de educação permanente para o manejo da tuberculose (CECÍLIO HPM, et al., 2018).

Assim, o baixo desempenho no indicador de capacitação está diretamente relacionado com a fragilidade da realização de ações educativas para às equipes de saúde, sendo os auxiliares/técnicos de Enfermagem e médicos as categorias mais afetadas (WYSOCKI D, et al., 2017).

As variáveis relacionadas aos registros de acompanhamento do paciente tiveram desempenho satisfatório, com exceção do acesso ao livro de registro e acompanhamento de ILTB que obteve desempenho insatisfatório. Os livros de registros permitem o acompanhamento da evolução do paciente em seu tratamento e seu desfecho, bem como analisar os resultados e a qualidade das ações realizadas na unidade de saúde (BRASIL, 2019).

O livro de registro e acompanhamento do tratamento da Infecção Latente da Tuberculose (ILTB) é uma importante ferramenta para o serviço de saúde e para a gestão, pois se faz necessário para as ações de vigilância epidemiológica e serve de instrumento para o cálculo de indicadores, tanto operacionais quanto epidemiológicos (BRASIL, 2014b).

A qualidade dos serviços de saúde é mensurada através da disponibilidade de recursos, insumos e humanos, além da organização do processo de trabalho. Desta forma, a APS tem um papel fundamental no tratamento ao paciente com tuberculose, já que é a principal porta de entrada do SUS e a mais resolutiva. Assim, o PNCT tem como estratégias a disponibilização, em tempo oportuno, de insumos para o diagnóstico de todas as formas de tuberculose: sensível, resistente e infecção latente (BRASIL, 2019). Os resultados obtidos neste estudo estão de acordo com o preconizado pelo MS em relação a disponibilidade de insumos como potes, pedidos de exames e fichas de referência e contrarreferência.

As variáveis entrega de medicamentos, acesso a consulta em $24 \mathrm{~h}$, orientação quanto a busca por atendimento fora da data agendada caso necessite e serviço laboratorial obtiveram desempenho satisfatório. Estes achados são corroborados por Brasil (2019) quando relata que os casos de eventos adversos deveram ser atendidos e notificados em qualquer unidade de saúde nas 24 horas após a sua ocorrência. Logo, é importante que a equipe de saúde oriente o paciente sobre o direito de buscar consulta fora da data estipulada em casos de necessidade. 
Ainda sobre as variáveis de organização do serviço, a única que obteve desempenho insatisfatório foi o acesso ao resultado do exame de baciloscopia de escarro em 7 dias. Portanto, o estabelecimento de rotinas e fluxos devem garantir o fluxo das amostras, retorno do resultado do TRM-TB e da baciloscopia em 24 horas para o início imediato do tratamento (BRASIL, 2019). Assim, os dados deste estudo refutam o que é preconizado pelo PNCT.

O componente processo foi o último componente a ser avaliado neste estudo e o que mais apresentou desempenho crítico nos resultados revelados revelando uma fragilidade no que diz respeito a esta categoria. Os itens que apresentaram desempenho crítico foram a atualização do livro de registro e acompanhamento, solicitação mensal da baciloscopia de controle, entrega do material na unidade para realização da baciloscopia e realização do PPD no mesmo serviço de saúde.

Entre as competências da atenção básica está o preenchimento correto e adequado dos dados nos diversos instrumentos de vigilância que são preconizados pelo PNCT. Dentre eles, destacamos o livro de registro e acompanhamento dos casos que deve ser preenchido adequado e estar sempre atualizado a fim de facilitar a organização do serviço e conhecer todos os casos acompanhados na unidade de saúde. Assim, foi possível identificar que o livro de registro não se encontra atualizado, dificultando a organização do serviço e indo contra a recomendação do PCNT (BRASIL, 2019).

Outro elemento vital no controle do tratamento da tuberculose é a baciloscopia de escarro. Esta importância não se limita apenas a confirmação da eficácia do tratamento, mas também tem o papel de avaliar os riscos para os contatos de pacientes bacilíferos. É recomendado que a baciloscopia de controle seja realizado mensalmente nos casos de TB pulmonar, sendo espero a negativação a partir do término da segunda semana de tratamento (BRASIL, 2019). Porém, neste estudo, observou-se que a solicitação mensal de baciloscopia apresentou desempenho crítico o que corrobora a necessidade de estratégias para a melhora desta variável.

Constatou-se, neste estudo, que a entrega do material para baciloscopia na própria unidade de saúde se dá de forma crítica, apresentando índices baixíssimos na avaliação dos profissionais entrevistados. A descentralização das ações constitui um desafio na APS sendo determinante para garantir o acesso ao diagnóstico precoce da doença. Além disto, estes autores tiveram o mesmo resultado obtido em seu estudo, sendo evidenciado a centralização de serviços e encaminhamento de exames para outras localidades (CECILIO HPM, TESTON EF, MARCON SS, 2017).

Ainda sobre a centralização de ações e serviços, a realização do PPD na unidade de saúde se deu de forma crítica. Muitos profissionais entrevistados não sabiam o que era o exame, seu objetivo e quando deveria ser solicitado. Outros afirmaram que o exame não era realizado na unidade e não sabiam informar onde o mesmo poderia ser efetuado. Este resultado é oposto às recomendações do PNCT, preconizando o diagnóstico precoce da ILTB pois seu diagnóstico e tratamento evitam adoecimentos da população com risco (BRASIL, 2019). Se a unidade não oferece este tipo de serviço, significa dizer que o cuidado integral está fragmentado e que existe um grande rico de subnotificações de ILTB na área abrangência da unidade Santos Dumont.

As demais variáveis do componente processo apresentaram resultados satisfatórios seja pelas orientações realizadas pelos profissionais aos pacientes, pela regularidade das medicações, a realização do teste de HIV, consultas mensais e busca ativa dos faltosos favorecendo a criação e fortalecimento do vínculo entre usuário, família e serviço de saúde, pois o paciente e sua família reconhecem o trabalho integral que a equipe de saúde dispõe para o seu cuidado (SILVA DM, et al., 2014).

\section{CONSIDERAÇÕES FINAIS}

Evidenciou-se que o serviço apresentou desempenho satisfatório na maioria das variáveis apresentadas, demonstrando um envolvimento com o PNCT e com o usuário pertencente aquele serviço, buscando ofertar um serviço de qualidade. Porém, em outras variáveis foram observadas algumas fragilidades apresentadas pelo serviço como: a alta rotatividade de profissionais médicos e enfermeiros, ponto este negativo para a 
criação de vínculo com o usuário do serviço; a falta de atualização do livro de registro e acompanhamento do paciente; a necessidade de capacitação constante sobre o manejo do tratamento e fluxos de serviços para o paciente e a dificuldade de se conseguir em tempo oportuno os resultados de baciloscopia. Para amenizar ou solucionar tais fragilidades faz-se necessário a adoção de um conjunto de estratégias com os diversos atores envolvidos no cenário de enfrentamento da TB, buscando uma assistência integral e criação de vínculos, levando em consideração as particularidades e o meio biopsicossocial do paciente e sua família.

\section{REFERÊNCIAS}

1. BARREIRA D. Os desafios para a eliminação da tuberculose no Brasil. Epidemiol. Serv. Saúde. Brasília, 2018; 27 (1).

2. BERTOLOZZI MR, et al. O controle da tuberculose: um desafio para a saúde pública. R. Med, 2014; 93 (2): 83-89.

3. BEZERRA LCA, et al. A vigilância epidemiológica no âmbito municipal: avaliação do grau de implantação das ações. Cad. Saúde Púb, 2009; 25 (4): 827-839.

4. BRASIL. Ministério da Saúde. Secretaria de Vigilância em Saúde. Departamento de Vigilância Epidemiológica. Manual de recomendações para o controle da tuberculose no Brasil. Brasília; 2a ed. atualizada, 2019.

5. BRASIL. Ministério da Saúde. Secretaria de Vigilância em Saúde. Departamento de Vigilância das Doenças Transmissíveis. Implantação do plano nacional pelo fim da tuberculose como problema de saúde pública no Brasil: primeiros passos rumo ao alcance das metas. Bol epidemiológico [Internet]. DF, V. 49, n. 11, p. 1-18, 2018a.

6. BRASIL. Ministério da Saúde. Secretaria de Vigilância em Saúde. Série Histórica do Número de Mortes por Tuberculose: Brasil, Regiões e Unidades Federadas de residência por ano do óbito (2001 a 2017). Brasília: Ministério da Saúde, 2018b.

7. BRASIL. Ministério da Saúde. Secretaria de Vigilância em Saúde. Brasil Livre da Tuberculose: Plano Nacional pelo Fim da Tuberculose como Problema de Saúde Pública. Brasília, 2017.

8. BRASIL. Ministério da Saúde. Secretaria de Vigilância em Saúde. Departamento de Vigilância das Doenças Transmissíveis. Panorama da tuberculose no Brasil: indicadores epidemiológicos e operacionais. Brasília, 2014a.

9. BRASIL. Ministério da Saúde. Secretaria de Vigilância em Saúde. Departamento de Vigilância das Doenças Transmissíveis. Livro de registro de pacientes e acompanhamento do tratamento da infecção latente da tuberculose [recurso eletrônico], Brasília: Ministério da Saúde, 2014b.

10. BRASIL. Ministério da Saúde. Secretaria de Vigilância em Saúde. Departamento de Vigilâncias das Doenças Transmissíveis. Experiências de monitoramento e avaliação no controle da tuberculose no Brasil. Brasília, 2013.

11. Brasil. Ministério da Saúde. Vigilância Epidemiológica. Manual de recomendações para o controle da tuberculose no Brasil. Brasília; 2011a.

12. Brasil. Ministério da Saúde. Secretaria de Vigilância em Saúde. Departamento de Vigilância Epidemiológica. Tratamento diretamente observado (TDO) da tuberculose na atenção básica: protocolo de enfermagem. Brasília, 2011b.

13. BRASIL. Ministério da Saúde. Ato Portaria GM n. 1.996, de 20 de agosto de 2007. Dispõe sobre as diretrizes para a implementação da Política Nacional de Educação Permanente em Saúde e dá outras providências. Brasília, 2007.

14. CECILIO HPM, FIGUEIREDO RM, MARCON SS. Coordenação e elenco de serviços no controle da tuberculose: percepção de enfermeiros e médicos. Cad. Saúde Colet. 2018; 26 (4): 439-445.

15. CECILIO HPM, TESTON EF, MARCON SS. Acesso ao diagnóstico de tuberculose sob a ótica dos Profissionais de saúde. Texto Contexto Enferm., 2017; 26 (3).

16. FELISBERTO $\mathrm{E}$, et al. Avaliação do processo de implantação da estratégia de Atenção Integrada às Doenças Prevalentes da Infância no Programa de Saúde da Família, no estado de Pernambuco, Brasil. Cad. de Saúde Públ., 2002; 18(6): 1737-1745. 
17. LIMA EFA. Perfil socioprofissional de trabalhadores de equipes saúde da família. Rev enferm UERJ, 2016; $24(1)$.

18. ROCHA MA. Acompanhamento do tratamento da tuberculose na Atenção Primária à Saúde: revisão integrativa. Trabalho de Conclusão de Curso (Bacharelado em Enfermagem) - Centro de Formação de Professores, Universidade Federal de Campina Grande, Cajazeiras, Paraíba, Brasil, 2016, 56f.

19. SAMICO I, et al. Atenção à saúde da criança: uma análise do grau de implantação e da satisfação de profissionais e usuários em dois municípios do estado de Pernambuco, Brasil. R. Bras. de Saúde Materno Infantil, 2005; 5 (2): 229-240.

20. SANCHO LG, et al. Rotatividade na força de trabalho da rede municipal de saúde de Belo Horizonte, Minas Gerais: um estudo de caso. Trab. Educ. Saúde, 2012; 9(3):431-447.

21. SILVA DM, et al. Tratamento da tuberculose na Atenção Básica: avaliação de estrutura e processo dos serviços de saúde. Dissertação de Mestrado em Enfermagem da Universidade Federal da Paraíba. João Pessoa, 2013.

22. SILVA DM, et al. Avaliação de desempenho de Serviços da Atenção Básica para o tratamento da tuberculose. Rev Esc Enferm USP, 2014; 48 (6): 1044-53.

23. TONELLI BQ, et al. Rotatividade de profissionais da Estratégia Saúde da Família no município de Montes Claros, Minas Gerais, Brasil. RFO UPF, 2018; 23 (2):180-185.

24. WORLD HEALTH ORGANIZATION. Global tuberculosis report 2018.

25. WYSOCKIL D, et al. Atenção Primária à Saúde e tuberculose: avaliação dos serviços. Rev Bras Epidemiol, 2017; 20 (1): 161-175. 